\title{
Investigation of Graphene Oxide in Diesel Soot
}

\author{
Swapna MS and Sankararaman $S^{*}$
}

Department of Optoelectronics, University of Kerala, Trivandrum, Kerala, India

${ }^{*}$ Corresponding author: Sankararaman S, Department of Optoelectronics, University of Kerala, Trivandrum, 695581, Kerala, India, E-mail: drssraman@gmail.com

Citation: Swapna MS, Sankararaman S (2017) Investigation of Graphene Oxide in Diesel Soot. J Mater Sci Nanotechnol 5(1): 103. doi: 10.15744/2348-9812.5.103

Received Date: May 04, 2017 Accepted Date: May 30, 2017 Published Date: June 02, 2017

\begin{abstract}
Graphene has emerged as a potential material in various scientific disciplines, ranging from material science, engineering, and more recently biomedicine. The paper describes the investigation of the presence of graphene and graphene oxide (GO) in the carbon soot of internal combustion diesel engines. The UV-Visible, Fourier transform infrared (FTIR), X-ray diffraction (XRD), Photoluminescent (PL) and Raman spectroscopic analysis of the sample provided a conclusive evidence of the formation of graphene and GO. The Field Emission Scanning Electron Microscopy (FESEM) and Energy Dispersive Spectrum (EDX) of the sample show carbon nanoparticles (CNPs) of size less than 50nm. The High -Resolution Transmission Electron Microscopy (HR-TEM) analysis confirmed the formation of graphene sheets with carbon nanospheres attached to it. The study reveals the possible exploitation of the diesel soot for potential applications in science and technology.
\end{abstract}

Keywords: Graphene; Graphene oxide; Carbon nanoparticles; Combustion; Carbon nanotube

List of Abbreviations: NGO: Graphene oxide; FTIR: Fourier transform infrared; XRD: X- ray diffraction; PL: Photoluminescent; FESEM: Field Emission Scanning Electron Microscopy; EDX: Energy Dispersive Spectrum; CNPs: Carbon nanoparticles; HR-TEM: High Resolution Transmission Electron Microscopy; 2D: Two-dimensional; GO: Graphene oxide; r- GO: Reduced graphene oxide; ICE: Internal combustion engines; G-band: Graphitic band; D band: Disorder band; RBM: Radial Breathing Mode; IFM: Intermediate Frequency Mode; oTO- LA: Out-of plane transverse optic-longitudinal acoustic modes

\section{Introduction}

Graphene, an allotrope of carbon, is a single-layer planar sheet of $\mathrm{sp}^{2}$ bonded carbon atoms that are packed in a two-dimensional honeycomb lattice. It is the thinnest and strongest free-standing two-dimensional (2D) basic structural element of other allotropes, including graphite, charcoal, carbon nanotubes and fullerenes. The mechanical strength, large conductivity, large surface area, and light weight make it a suitable material for high-capacity energy storage batteries. Intrinsic graphene behaves like a semi-metal or zero-gap semiconductor. It can be considered as an indefinitely large aromatic molecule, the ultimate case of the family of flat polycyclic aromatic hydrocarbons. Its unique honeycomb carbon geometry makes it a potential material in various scientific disciplines, ranging from material science, engineering and more recently biomedicine [1-8].

A bulk solid made by oxidation of graphite with increased interlayer spacing is termed as graphite oxide. Chemically modified graphene formed as a byproduct of this oxidation process is called graphene oxide (GO). Reducing graphene oxide by various physical, thermal and chemical methods produce reduced graphene oxide ( $r$ - GO) which is commonly employed for producing large quantities of good quality graphene for various industrial applications. Based on the number of well- defined countable stacked layers there are the bilayer, tri-layer, multi-layer and few-layer graphene. The single-atom-thick sheet of carbon atoms having a thickness or lateral dimension less than 100nm are classified as Graphene nanoplates, nanosheets, nano- flakes [9]. The most commonly used bottom-up and top-down methods for the synthesis of graphene and its derivatives are chemical vapor deposition, micromechanical exfoliation, solvent stripping, ball milling, oxidative functionalization etc.

Nowadays the world is worried about the pollution due to old vehicles and internal combustion engines. The present work is an attempt to investigate the formation of GO by the incomplete combustion of the hydrocarbon- diesel. In internal combustion engines (ICE), combustion of the fuel, diesel takes place at high temperature producing carbon nanoparticles. The high-temperature combustion may lead to the formation of graphene sheets containing carbon nanotubes and graphene oxide [10]. Lesser the efficiency of ICE greater is the possibility of formation of particulate matter in addition to carbon dioxide and water. Soot particles are solid, carbon-rich $(\sim 98 \%)$ material formed by the vapor phase condensation as a product of combustion [11]. Thus the study is helpful in turning the hazardous diesel soot into useful material for super capacitive energy storage applications. 


\section{Materials and Methods}

The soot particles formed by the incomplete combustion of diesel from ICE are collected and purified by liquid phase oxidation method. Purification of the carbonaceous soot particles are essential since the impurities in them may affect the properties of the nanotubes, graphene layers etc. present in the sample. In the liquid phase oxidation method the sample is mixed with sulphuric acid and nitric acid in the ratio 1:3, ultrasonicated using Scientech SE-366 for 20 minutes, filtered with Whatman Filter paper 41 and washed with distilled water four times. Then it is quenched with ice-cooled water and base neutralized with sodium hydroxide [12]. Then the sample is again washed with distilled water four times and filtered with Whatman Filter paper 42. The purified sample is subjected to morphological characterization by Nova Nano FESEM and JEOL JEM- 2100 TEM. The composition study is carried out by EDX. XRD measurements are done in Bruker D8 Advanced Diffractometer with CuKa radiation ( $\lambda=1.5406 \AA$ ). The functional groups are identified by FTIR which is recorded using Shimadzu IR Prestige-21 and Raman spectrum using Lab Ram Micro-Raman Spectrometer with Argon ion laser (at $514.5 \mathrm{~nm}$ wavelength and power of $5 \mathrm{~mW}$ ) as the excitation source. UVVisible spectrum is recorded using Jasco V 550 UV-Visible spectrophotometer and Photoluminescent Spectrum of the sample is recorded using Horiba Fluoromax.

\section{Results and Discussions}

The FESEM image of the carbon particles formed from the internal combustion diesel engine is shown in Figure 1(a). It indicates spherical carbon nanoparticles with size less than $50 \mathrm{~nm}$. Elemental analysis by EDX spectra of the sample shows the presence of carbon, oxygen, sodium, sulphur, calcium and potassium. The EDX spectrum of carbonaceous diesel soot is shown in Figure 1(b). The HR-TEM images of the sample are shown in Figure 2. The TEM images confirm the formation of graphene sheets with carbon nanospheres attached to it. The image shows the multi-layered graphene sheets.
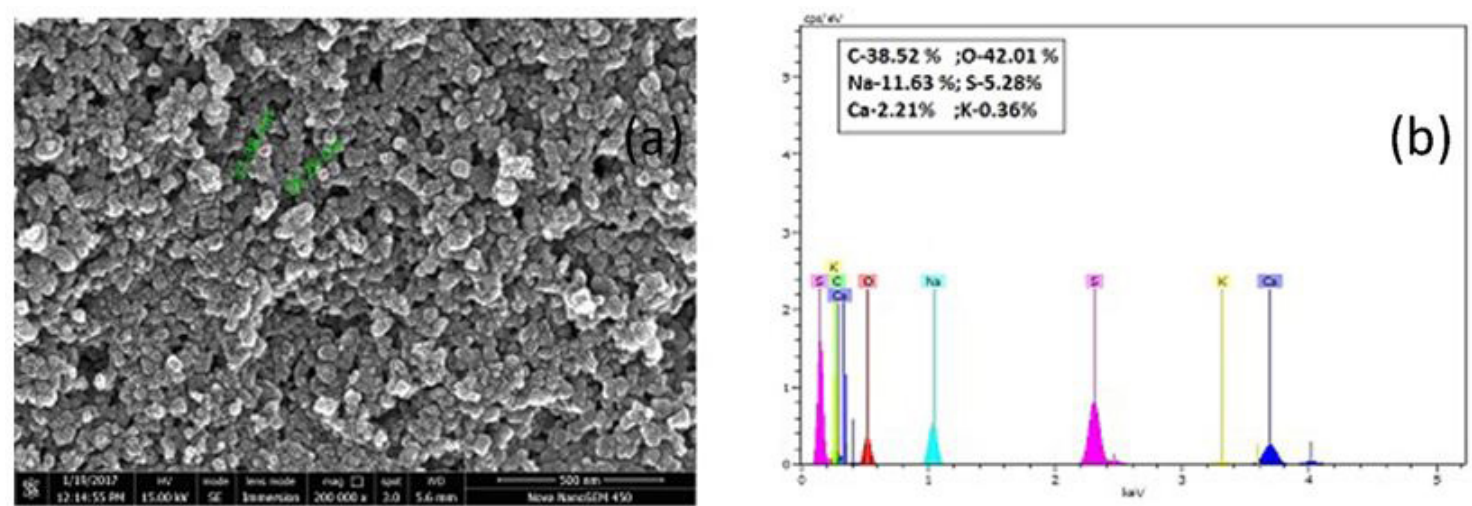

Figure 1: (a) FESEM image (b) EDX spectra of the sample
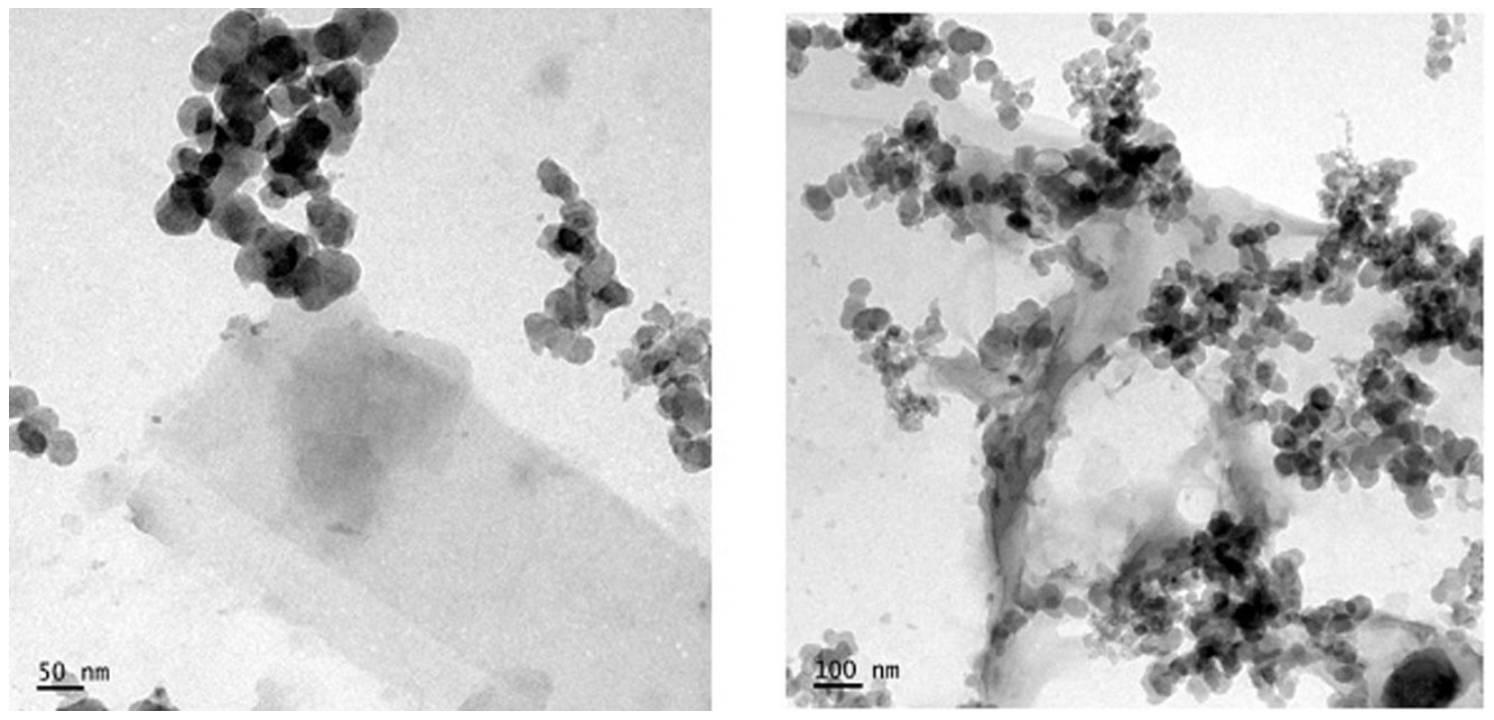

Figure 2: HR-TEM images of graphene sheets with carbon nanospheres attached

The UV-Visible absorption spectrum gives the electronic transitions from the ground state to the excited state. The spectrum shows a peak at $247 \mathrm{~nm}$ that arises from the $\pi-\pi^{\star}$ transition of $\mathrm{C}-\mathrm{C}$ and $\mathrm{C}=\mathrm{C}$ bonds in the $\mathrm{sp}^{2}$ hybrid region. The peaks at 278 $290 \mathrm{~nm}$ region is due to the $\mathrm{n}-\pi^{*}$ transition of the $\mathrm{C}=\mathrm{O}$ bond of the $\mathrm{sp}^{3}$ hybrid region $[13,14]$. This type of absorption spectrum is reported to be exhibited by graphene comprising a single layer of carbon atoms [15]. The UV- Visible absorption spectrum of the sample is shown in Figure 3(a). 

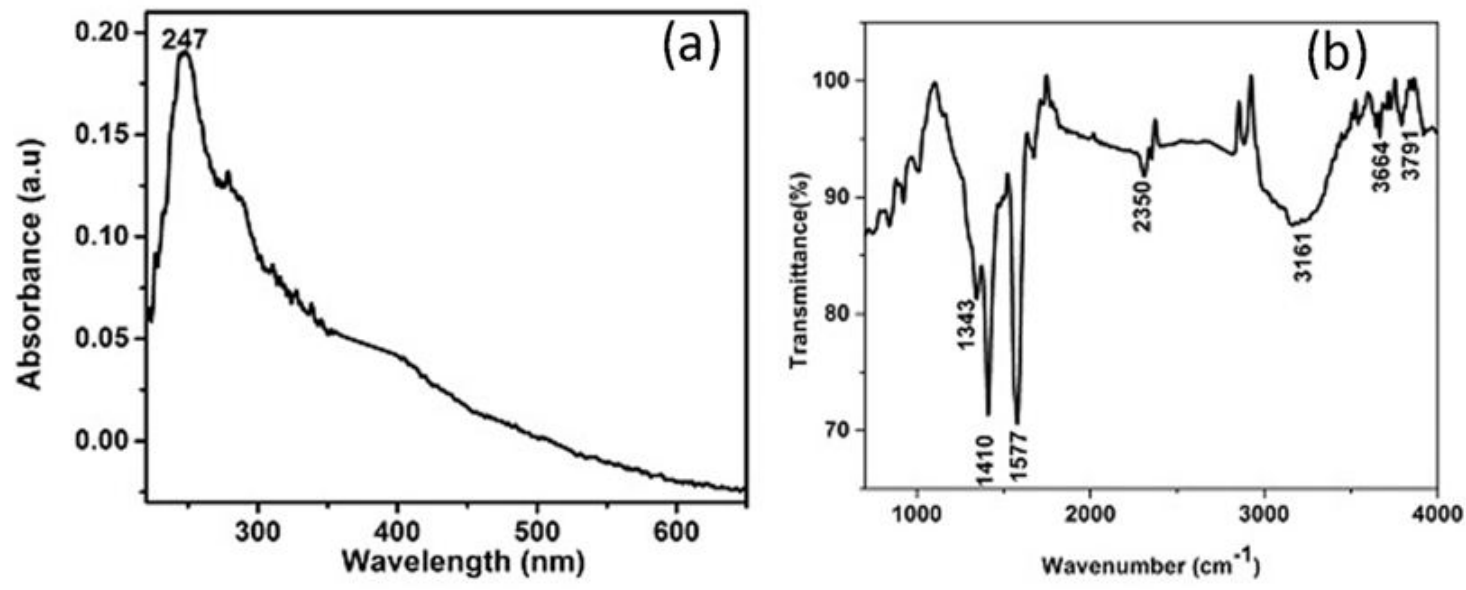

Figure 3: (a) UV- Visible spectrum (b) FTIR of the sample

The identification of the surface functional groups is done with the FTIR spectroscopy. The FTIR spectrum of the sample is shown in Figure 3(b). Graphene oxide is considered as the precursor for graphene synthesis. It contains various functional groups including $\mathrm{C}-\mathrm{O}, \mathrm{C}=\mathrm{O},-\mathrm{OH},-\mathrm{C}-\mathrm{O}-\mathrm{C}$ that have a significant role in the properties exhibited by the graphene oxide sheets [16,17]. The peak around 1343 and $1410 \mathrm{~cm}^{-1}$ is attributed to the deformation of $\mathrm{CH}_{3}$ and $\mathrm{CH}_{2}$ groups [1]. The region $1500-1650 \mathrm{~cm}^{-1}$ is said to be the aromatic region, in which peak at $1577 \mathrm{~cm}^{-1}$ may be due to the double bonds with one substitution [18,19]. The region 2500-3650 $\mathrm{cm}^{-1}$ corresponds to $-\mathrm{OH}$ stretching vibrations and the presence of aromatic and unsaturated bonds present in the sample $[15,20]$. The FTIR spectrum of the sample under investigation shows the characteristic peaks of graphene as evidenced by literature. This gives an indication of the possibility of the presence of graphene in the sample.

Photoluminescent (PL) spectroscopy is a contactless, nondestructive method for getting information about the electronic structure [21]. The Photoluminescent Spectra of the sample are recorded for three different excitation wavelengths 350nm, 430nm, and $510 \mathrm{~nm}$. The corresponding emission spectra obtained in the range 400-800nm are shown in Figure 4 (a), (b) and (c).
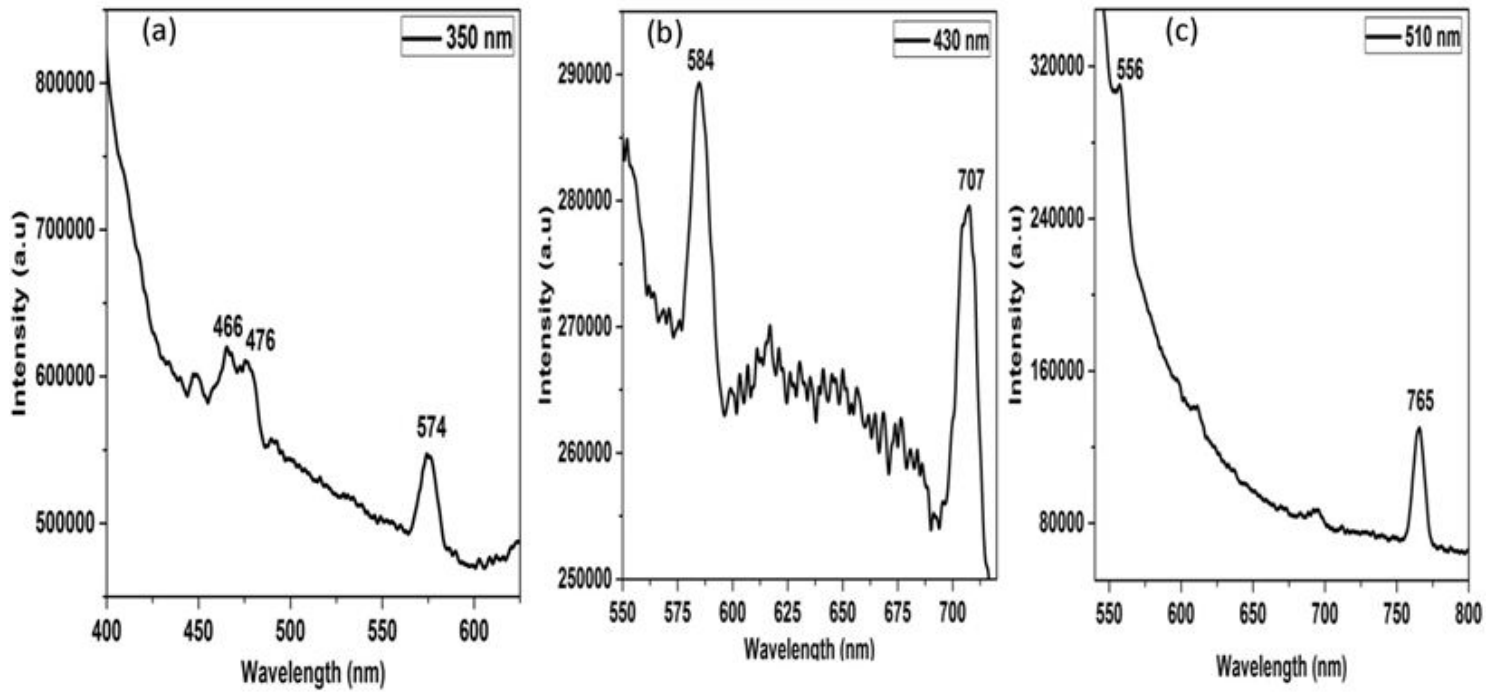

Figure 4: PL Spectra (a) $\lambda_{e x}=350 \mathrm{~nm}\left(\right.$ b) $\lambda_{e x}=430 \mathrm{~nm}$ (c) $\lambda_{e x}=510 \mathrm{~nm}$

It can be seen that as the excitation wavelength is increased from $350 \mathrm{~nm}$ to $510 \mathrm{~nm}$, the maximum emission is shifted to the longer wavelength region. From literature it can be seen that the broad emission band in the region $400-800 \mathrm{~nm}$ is exhibited by the PL spectra of GO [22]. The PL spectra of the sample also indicate a similar nature and thereby revealing the presence of GO. This type of redshift is an indicative of combined aromatic groups or cyclic molecules with several pi $(\pi)$ bonds [22]. The fluorescence exhibited by the graphene oxide may be due to the optical transitions occurring in the $\pi-\pi^{*}$ gap of $\mathrm{sp}^{2}$ sites [23,24]. The role of $\mathrm{sp}^{2}$ clusters within the $\mathrm{sp}^{3}$ matrix is very prominent in deciding the emission wavelengths. Thus the PL spectra of the sample provide an information about the presence of GO which is in agreement with the FTIR spectra.

A color emission from the sample on photoexcitation are measured and expressed in terms of the resultant chromaticity coordinates $(x, y)$. Taking the International Commission on Illumination (CIE) XYZ color space as the standard reference the chromaticity diagram of the sample is plotted for the three different excitations at 350nm, 430nm, and 510nm and is shown in Figure 5. The $x$ and ${ }^{y}$ chromaticity coordinates for the three excitation wavelengths are calculated in the CIE XYZ color space. For the excitation at $350 \mathrm{~nm}, 430 \mathrm{~nm}$ and $510 \mathrm{~nm}$ the emissions are obtained at the CIE coordinates $(0.321,0.313)$ in the bluish pink region, $(0.272,0.280)$ in the purplish blue region and $(0.424,0.567)$ in the yellowish green region. 


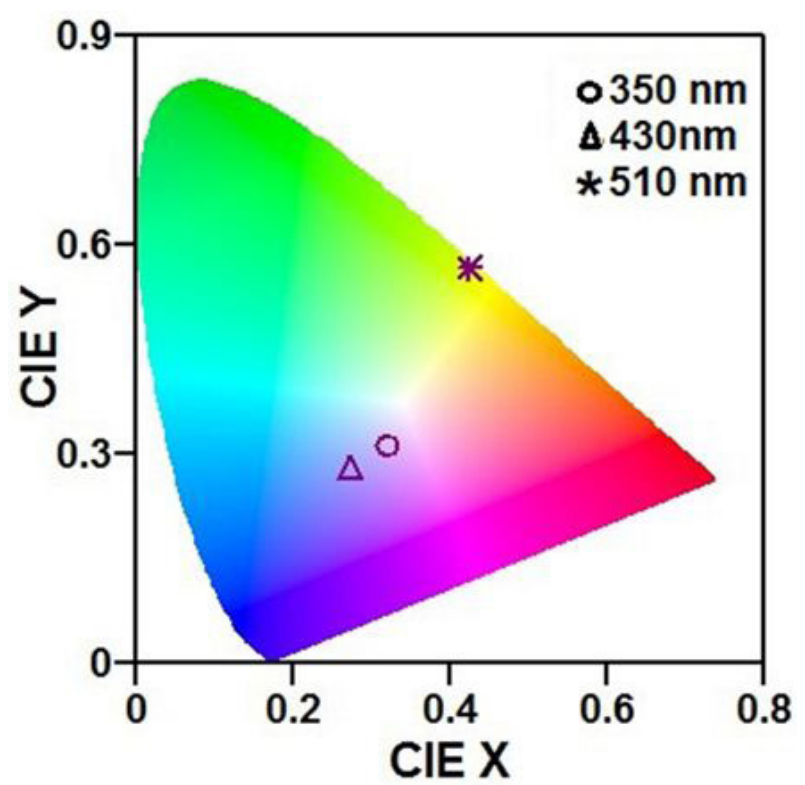

Figure 5: CIE plots of the sample at different excitation wavelengths

The XRD spectrum of the sample is shown in Figure 6(a). On deconvolution of the spectra, a small peak can be seen in the range $10-15^{\circ}$, which is a characteristic of GO. The two-theta values at $25.17^{\circ}$ and $42.96^{\circ}$ are identified as graphite peaks corresponding to planes (002) and (101). The presence of these peaks suggests the formation of multi-layered GO sheets due to the strong interactions existing between the layers. This further confirms the presence of GO in the sample.
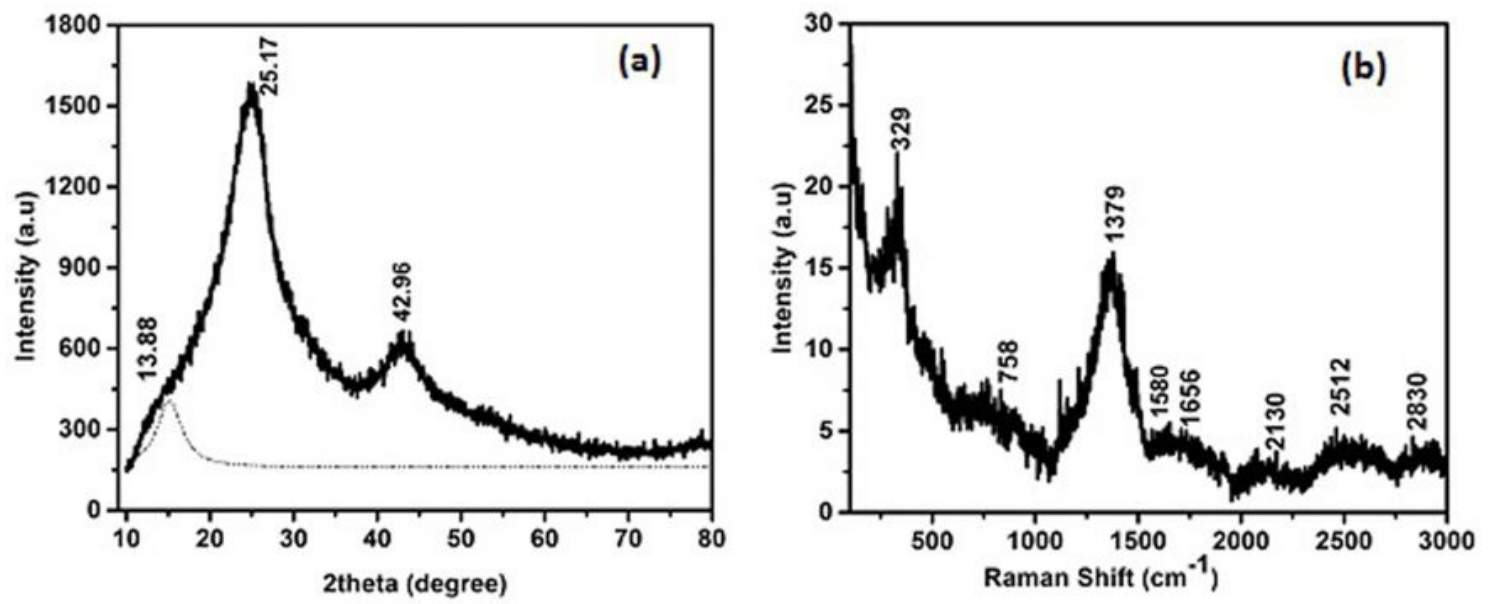

Figure 6: (a) XRD pattern (b) Raman spectra of the sample

Raman spectroscopy is an efficient non-destructive tool for the characterization of all types of carbon allotropes. This technique is one among the most reliable methods to determine the quality of graphene by understanding the defect density within the $\mathrm{C}$-framework [1]. It gives various information about the $\mathrm{sp}^{2}$ hybridized nano carbons which includes graphene and graphenerelated materials, carbon nanotubes etc. [25]. The Raman spectrum of the sample is shown in Figure 6(b). The Raman peaks observed and its assignments are given in Table 1. The presence of D peak, G and 2D (or G') peaks at 1379, 1580 and 2400-3250 $\mathrm{cm}^{-1}$ respectively are the characteristic peaks exhibited by graphene. The $\mathrm{G}$ band corresponds to the first order scattering of the $\mathrm{E}_{2 \mathrm{~g}}$ stretching vibration mode of $\mathrm{sp}^{2}$ carbon and is an indication of phonon emission around $1580 \mathrm{~cm}^{-1}$.

The $\mathrm{D}$ peak shows the disorder in the crystal structure whereas the $2 \mathrm{D}$ peak indicates the graphene structure. If the defect density is about $1-3 \%$ then the $2 \mathrm{D}$ peak is only barely detectable (no sharp peak). The low intensity of $2 \mathrm{D}$ peak shows that the structure of graphene is destroyed and a considerable amount of defects are introduced within the carbon framework as a result of the formation of graphene [28]. Figure 5 also exhibit the same nature with low-intensity 2D peak indicating the formation of graphene.

The defect density can be evaluated from the ratio of $\mathrm{I}_{\mathrm{D}} / \mathrm{I}_{\mathrm{G}}$ or $\mathrm{I}_{2 \mathrm{D}} / \mathrm{I}_{\mathrm{G}}$ ratio and in the present case, it is found to be $\sim 3$ which is in agreement with the literature [22]. The planar microcrystallite size $\left(\mathrm{L}_{\mathrm{a}}\right)$ for the samples are calculated from the intensities of $\mathrm{D}$ and $\mathrm{G}$ bands of Raman spectra using empirical relation $\mathrm{L}_{\mathrm{a}}=\left(102^{*}\left(\mathrm{I}_{\mathrm{G}} / \mathrm{I}_{\mathrm{D}}\right)\right)^{0.5} \mathrm{~nm}$ [29]. The crystallite size is found to be $\sim 5 \mathrm{~nm}$, for the Raman excitation wavelength at $514.5 \mathrm{~nm}$. 


\begin{tabular}{|c|c|c|}
\hline Raman Shift $\left(\mathrm{cm}^{-1}\right)$ & Vibration Modes & Spectral assignments \\
\hline 329 & $\begin{array}{l}\text { RBM (Radial Breathing } \\
\text { Mode) }\end{array}$ & Nanotube only, vibration of radius [26] \\
\hline 758 & $\begin{array}{l}\text { IFM (Intermediate Fre- } \\
\quad \text { quency Mode) }\end{array}$ & $\begin{array}{l}\text { Combination mode oTO-LA [26] (out - } \\
\text { of plane transverse optic - longitudinal } \\
\text { acoustic mode) }\end{array}$ \\
\hline 1379 & $\mathrm{D}$ band & $\begin{array}{c}\text { Double resonance effects in } \mathrm{sp}^{2} \text { carbon } \\
\text { [27] Attributable to the presence of } \\
\text { graphene }\end{array}$ \\
\hline 1580 & $\mathrm{G}^{-}$band & $\begin{array}{l}\text { Raman active mode of graphite [26] At- } \\
\text { tributable to the presence of graphene }\end{array}$ \\
\hline 1656 & G+ band & Raman active mode of graphite [26] \\
\hline 2130 & $\mathrm{M}^{-}$band & Overtone of oTO mode [26] \\
\hline 2512 & $\mathrm{G}^{\prime}$ band & $\begin{array}{l}\text { Overtone of D mode }[24,27] \\
\text { Attributable to the presence of graphene }\end{array}$ \\
\hline 2830 & G'band & $\begin{array}{l}\text { Overtone of D mode }[24,27] \\
\text { Attributable to the presence of graphene }\end{array}$ \\
\hline
\end{tabular}

Table 1: Raman spectral assignments

Graphene exhibits two hexagonal structures such as zigzag and armchair. It is well reported in the literature that the strong D peak compared to the weak $G$ peak arises from the armchair structure [30,31]. Figure 6(b) is in agreement with the reports in the literature. These peaks along with UV, FTIR, PL and XRD data provide a conclusive evidence for the existence of graphene in the diesel soot from ICE. The TEM images provide the direct proof for the formation of graphene sheets.

\section{Conclusion}

The study reveals the possible exploitation of the diesel soot for potential applications in science and technology and suggests a solution for the worry of the world about the pollution due to old vehicles and internal combustion engines. The analysis of the purified samples from the ICE reveals the formation of graphene oxide and opens a new window for the effective use of the soot particles in fuel cell, nanocapacitors, etc. The HR-TEM and FESEM analysis show multi- layered graphene sheets with carbon nanospheres of size less than 50nm and the EDX revealed the richness of carbon in the sample. The structural (FTIR, XRD, and Raman) and optical (UV and PL) characterizations of the particulate matter collected from the internal combustion diesel engines are found to exhibit the existence of graphene. Thus the hazardous diesel soot can be converted into an useful material for electronic applications.

\section{References}

1. Nazarpour S, Waite SR (2016) Graphene Technology: From Laboratory to Fabrication. Wiley- VCH, Germany.

2. Vikrant S, Monu M, Govind G, Gurmeet S, Sharma RK (2017) Turning Hazardous Diesel Soot into High Performance Carbon/MnO 2 Supercapacitive Energy Storage Material. ACS Sustainable Chem Eng 5: 450-9.

3. Avouris P, Dimitrakopoulos C (2012) Graphene: synthesis and applications. Mater Today 15: 86-97.

4. Eduardo RH, Margarita D, Fernandes FM, Ezzouhra Z, Palomares FJ, et al. (2011) Supported Graphene from Natural Resources: Easy Preparation and Applications. Adv Mater 23: 5250-5.

5. Zhan B, Li C, Yang J, Jenkins G, Huang W, et al. (2014) Graphene Field-Effect Transistor and Its Application for Electronic Sensing. Small 10: $4042-65$.

6. Novoselov KS, Geim AK, Morozov SV, Jiang D, Zhang Y, et al. (2004) Electric field effect in atomically thin carbon films. Science 306: 666-9.

7. Geim AK, Novoselov KS (2007) The rise of graphene. Nature Mater 6: 183-91.

8. Balandin AA, Ghosh S, Bao W, Calizo I, Teweldebrhan D, et al. (2008) Superior thermal conductivity of single-layer graphene. Nano Lett 8: $902-07$.

9. Bianco A ( 2013 ) All in the graphene family - A recommended nomenclature for two-dimensional carbon materials. Carbon 65: 1-6.

10. Ermakov VA, Alaferdov, Vaz AR, Perim E, Autreto PAS, et al. (2015) Burning Graphene Layer-by-Layer. Scientific Reports 5: 11546.

11. Buseck PR, Adachi K, Gelencser A, Tompa E, Posfai M (2012) Are black carbon and soot the same? Atmos Chem Phys Discuss 12: $24821-46$.

12. Hussain S, Jha P, Chouksey A, Raman R, Islam SS, et al. (2011) Spectroscopic investigation of modified single wall carbon nanotube (SWCNT). J Mod Phys 2: 538-43.

13. Cuong TV, Phama VH, Tranb QT, Hahnc SH, Chunga JS, et al. (2010) Photoluminescence and Raman studies of graphene thin films prepared by reduction of graphene oxide. Mater Lett 64: 399-401.

14. Luo Z, Lu Y, Somers LA, Johnson AT (2009) High yield preparation of macroscopic graphene oxide membranes. J Am Chem Soc 131: 898-9.

15. Jasim DA, Lozano N, Kostarelos K (2016) Synthesis of few-layered, high-purity graphene oxide sheets from different graphite sources for biology. IOP Publishing 2D Mater. 3: 014006.

16. Stoller MD, Park S, Yanwu Z, An J, Ruoff RS (2008) Graphene-based ultracapacitors. Nano Letters 8: 3498-502.

17. Dreyer DR, Park S, Bielawski CW, Ruoff RS (2010) The chemistry of graphene oxide. Chemical Society reviews 39: 228-40.

18. Wang G, Wang B, Park J, Yang J, Shen X, et al. (2009) Synthesis of enhanced hydrophilic and hydrophobic graphene oxide nanosheets by a solvothermal method. Carbon 47: 68-72. 
19. Williams DH, Feleming I (1995) Spectroscopic Methods in Organic Chemistry. 5th edition. McGraw-Hill, England.

20. Gómez I, Mejía E, Cabanzo R (2013) Synthesis of graphene oxide and chemically reduced graphene nanosheets. Revista Colombiana de Mater 5: 177-84.

21. Andrew R, Barron R (1999) Photoluminescence Spectroscopy and its Application. Rice University.

22. Subach FV, Verkhusha VV (2012) Chromophore transformations in red fluorescent proteins. Chem Rev 112: 4308-27.

23. Chien CT, Li SS, Lai WJ, Yeh YC, Chen HA, et al. (2012) Tunable photoluminescence from graphene oxide. Angew Chem Int Ed Engl 51: 1-6.

24. Eda G, Lin YY, Mattevi C, Yamaguchi H, Chen HA, et al. (2010) Blue photoluminescence from chemically derived graphene oxide. Adv Mater 22: 505-9.

25. Ferrari AC, Robertson J (2004) Raman spectroscopy of amorphous, nanostructured, diamond-like carbon, and nano diamond. Philos. The Royal Society 362: 2477-512.

26. Dresselhaus MS, Dresselhaus G, Saito R, Jorio A (2005) Raman spectroscopy of carbon nanotubes. Physics Reports 409: 47-99.

27. O'Connell MJ, Bachilo SM, Huffman CB, Moore VC, Strano MS, et al. (2002) Band gap fluorescence from individual single-walled carbon nanotubes. Science 297): 593-6.

28. Lucchese MM, Stavale F, Ferreira EH. Vilane C, Moutinho MVO, et al. (2009) Quantifying ion-induced defects and Raman relaxation length in graphene. Carbon 48: 1592-97.

29. Childres I, Jauregui LA, Park W, Caoa H, Chen YP (2013) Raman spectroscopy of graphene and Related materials.

30. Cancado LG, Pimenta MA, Neves BRA, Dantas MSS, Jorio (2004) Influence of the Atomic Structure on the Raman Spectra of Graphite Edges. Phys Rev Lett 93: 247401.

31. You Y, Ni ZH, and Shen ZX (2008) Edge chirality determination of graphene by Raman spectroscopy. Appl Phys Lett $93: 163112$.

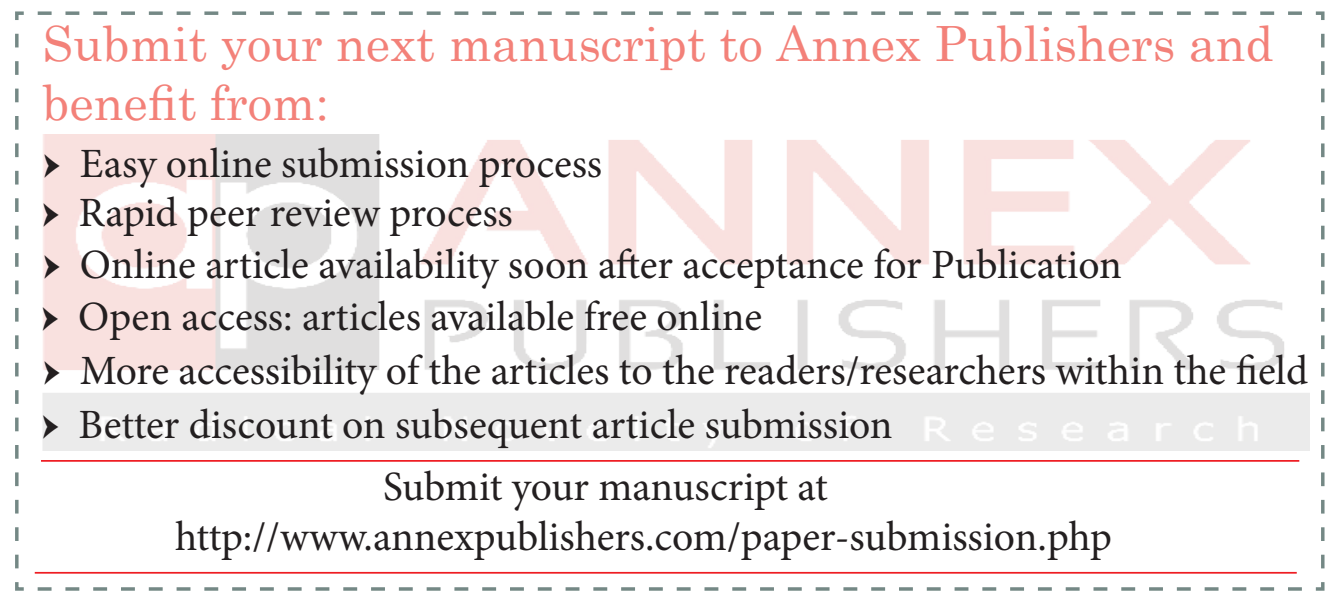

